\title{
On a question of Sierpiński
}

\author{
by
}

Theodore A. S laman (Berkeley, Ca.)

\begin{abstract}
There is a set $U$ of reals such that for every analytic set $A$ there is a continuous function $f$ which maps $U$ bijectively to $A$.
\end{abstract}

1. Introduction. A set of real numbers is analytic if it is a continuous image of a Borel set. It is projective if it can be obtained from a Borel set by a finite iteration of complementation and application of continuous functions.

Initially, the projective sets were studied topologically. Much of the progress was limited to the analytic sets, for which a variety of regularity properties were established. Among many, we should mention Borel, Luzin, Novikov, Sierpiński, and Suslin as having made significant contributions; see [2] for a more thorough discussion.

We will make use of Suslin's Perfect Set Theorem. Recall a perfect set is a nonempty closed set with no isolated points.

Theorem 1.1 (Suslin; see [3]). Every uncountable analytic set has a perfect subset.

It follows that any uncountable analytic set has the same cardinality as the continuum.

Our discussion will not be sensitive to variations in topology, but it will be most convenient for us to work with the Baire space ${ }^{\omega} \omega$, the set of $\omega$-sequences of natural numbers. In what follows, we construct a point in the Baire space as a real number.

We can restate Theorem 1.1 in the following way. For every analytic subset $A$ of ${ }^{\omega} \omega$ there is a subtree $T$ of ${ }^{<\omega} \omega$, the set of finite sequences of natural numbers, such that $T$ has no terminal nodes, every node in $T$

1991 Mathematics Subject Classification: 03E50, 04A15.

Key words and phrases: Borel set, analytic set.

The author wishes to thank Marcia J. Groszek for her simplification to the original proof of Theorem 2.3. The author was partially supported by National Science Foundation Grant DMS-9500878. 
has incompatible extensions in $T$, and every infinite path through $T$ is an element of $A$. Such a tree is called a perfect tree. When $T$ is a perfect tree we use $[T]$ to denote the set of infinite paths through $T$. We note that if $T$ is perfect, then there is a continuous injection of ${ }^{\omega} \omega$ into $[T]$. For example, one could map $\left(n_{1}, n_{2}, \ldots\right)$ first to the binary sequence $b$ consisting of $n_{1}$ many 1's, a $0, n_{2}$ many 1 's, a 0 , and so forth. Then map $b$ to the element $t$ of $[T]$ obtained by recursion: when for the $k$ th time we must decide a value for $b$ and there is more than one possibility in $T$, we take the least possible value if the $k$ th element of $b$ is 0 and take the next to least possible value otherwise. We will simply say that any $Z$ in ${ }^{\omega} \omega$ canonically determines an element of $[T]$.

These classical notions have natural syntactic formulations. For example, $A$ is analytic if membership in $A$ is definable by

$$
X \in A \Longleftrightarrow(\exists W)(\forall n) R(n, X\lceil n, W\lceil n, Z\lceil n)
$$

where $R$ is a recursive predicate, $Z$ is a fixed real parameter, $W$ ranges over the real numbers, $n$ ranges over the natural numbers, and $\lceil n$ denotes the restriction of a real to its first $n$ values. In (1.1), we say that membership in $A$ has been defined by a formula that is $\Sigma_{1}^{1}(Z): \Sigma$ because the formula begins with an existential quantifier, 1 in the superscript because the highest order quantifiers range over the real numbers, 1 in the subscript because there is only one alternation in the real quantifiers, and with the argument $Z$ because $Z$ appears as a parameter in the definition. We write $\boldsymbol{\Sigma}_{1}^{1}$ to denote the collection of analytic sets, that is, the collection of sets that are $\Sigma_{1}^{1}(Z)$ for some $Z$. Now we use recursion to define the projective hierarchy. We say that a set is $\boldsymbol{\Pi}_{n}^{1}$ if its complement is $\boldsymbol{\Sigma}_{n}^{1}$. We say that a set $S$ is $\boldsymbol{\Sigma}_{n+1}^{1}$ if membership in $S$ can be defined by $X \in S \Leftrightarrow(\exists W) R(X, W)$, where $R$ is $\boldsymbol{\Pi}_{n}^{1}$. A set that is both $\boldsymbol{\Sigma}_{n}^{1}$ and $\boldsymbol{\Pi}_{n}^{1}$ is said to be $\boldsymbol{\Delta}_{n}^{1}$. At the base of the projective hierarchy, Suslin showed that a set of reals is $\boldsymbol{\Delta}_{1}^{1}$ if and only if it is Borel. Globally, a set is projective if and only if it is $\boldsymbol{\Sigma}_{n}^{1}$ for some $n$. It was Luzin who showed that the projective hierarchy is a proper hierarchy: for each $n, \boldsymbol{\Delta}_{n}^{1} \varsubsetneqq \boldsymbol{\Sigma}_{n}^{1}$.

The fact that the classical descriptive set theorists were often limited to the analytic sets was well explained by Gödel.

TheOREM 1.2 (Gödel [1]). The set of constructible reals is $\Sigma_{2}^{1}$ and has a $\Sigma_{2}^{1}$ wellordering.

Consequently, if every real number is constructible (as is consistent with ZFC, the standard axioms of set theory), then there is a projective set with no perfect subset and so forth.

Modern descriptive set theory, in particular the theory of the projective sets, is well integrated with axiomatic set theory (in a strikingly beautiful 
way). For the most part, independence theorems show that the classical descriptive set theorists proved the strongest theorems possible, given that they were working within ZFC.

In this paper, we reverse the metamathematical trend toward axiomatic independence, and work entirely within ZFC. On the other hand, we apply intuitions of modern mathematical logic. We consider the following problem.

Problem 1.3 (Sierpiński [5]). Does there exist a set $U$ of reals such that for every uncountable analytic set $A$, there is a continuous function $f$ that maps $U$ bijectively to $A$ ?

It is not difficult to find a set $U$ such that every uncountable analytic set $A$ is a continuous image of $U$. There is even an analytic example. But these examples do not satisfy the requirement that continuous functions be injective on $U$.

We answer Problem 1.3 in two parts. First, no analytic set can have the required property. Second, there is a set $U$ as required, constructed using a wellordering of the reals.

\section{Solution to Sierpiński's problem}

2.1. No analytic solution. We begin with the easier calculation that there is no analytic solution to Problem 1.3. We apply a classical theorem.

Theorem 2.1 (Luzin [3]). Suppose that B is a Borel subset of $\omega_{\omega} \omega$, and that $f$ is a continuous function that is injective on $B$. Then the range of $f$ applied to $B$ is a Borel set.

Corollary 2.2. Suppose that $U$ is an analytic set. Then there is an uncountable analytic set $A$ such that there is no continuous bijection from $U$ to $A$.

Proof. Suppose to the contrary that for each uncountable analytic set $A$, there is a continuous bijection from $U$ to $A$. In particular, there is a continuous bijection $f$ from $U$ to ${ }^{\omega} \omega$. But then for each $X \in{ }^{\omega} \omega$ and for all $n$ and $m$ in $\omega$,

$$
f^{-1}(X)(n)=m \Leftrightarrow(\exists Z)[Z \in U \text { and } Z(n)=m \text { and } f(Z)=X] .
$$

Thus, for each $X$, the set of natural numbers $f^{-1}(X)$ is uniformly $\Sigma_{1}^{1}(X, f)$, and similarly uniformly $\Delta_{1}^{1}(X, f)$. Consequently, for all $Y, Y$ is in $U$ if and only if $Y$ is equal to $f^{-1}(f(Y))$, and so $U$ is $\Delta_{1}^{1}$, and therefore Borel.

Now, for every uncountable analytic set $A$, there is a continuous function that is injective on the Borel set $U$ and has range $A$. By Theorem 2.1, every uncountable analytic set is Borel, contradicting $\boldsymbol{\Delta}_{1}^{1} \neq \boldsymbol{\Sigma}_{1}^{1}$.

2.2. Constructing a solution-first approximation. Let $2^{\omega}$ be the cardinality of the continuum. (So, $2^{\omega}$ is an ordinal.) We begin by giving a heuristic 
construction of a one-parameter family of functions, each mapping a subset of $2^{\omega}$ bijectively to an associated $\boldsymbol{\Sigma}_{1}^{1}$ subset of ${ }^{\omega} \omega$. Later, we will replace $2^{\omega}$ by a set of reals.

Let $<$ be a wellordering of the finite sequences from ${ }^{\omega} \omega$. We may assume that $<$ has length $2^{\omega}$. Fix an indexing of the analytic sets by reals. For each real number $Z$, let $A(Z)$ denote the analytic set specified by $Z$.

We define the function $F(\gamma, Z)$ by transfinite recursion on $\gamma \in 2^{\omega}$. To specify the recursion step, let $\left(Y_{\gamma}, Z_{\gamma}\right)$ be the <-least pair such that $Y_{\gamma} \in$ $A\left(Z_{\gamma}\right)$ and $Y_{\gamma}$ is not in the range of $F\left(-, Z_{\gamma}\right)$ applied to ordinals less than $\gamma$. If $Z$ is equal to $Z_{\gamma}$, then let $F(\gamma, Z)$ equal $Y_{\gamma}$. Otherwise, let $F(\gamma, Z)$ be an element of $A(Z)$ which is not in the range of $F(-, Z)$ applied to ordinals less than $\gamma$, if there is such an element.

If $A(Z)$ is countable, then the domain of $F(-, Z)$ is some countable ordinal depending on the construction. If $A(Z)$ is not countable, then it has cardinality $2^{\omega}$ and the domain of $F(-, Z)$ is $2^{\omega}$.

Clearly, for each $Z, F(-, Z)$ is an injective function.

For a contradiction, suppose that there are $Y^{*}$ and $Z^{*}$ with $Y^{*}$ in $A\left(Z^{*}\right)$ but not in the range of $F\left(-, Z^{*}\right)$, and let $(Y, Z)$ be the least pair with this property. Let $\beta$ be the ordinal height of $(Y, Z)$ in $<$. Then, before or during the $\beta$ th step of the construction, $(Y, Z)$ must appear in the definition of $F$. At the stage during which $(Y, Z)$ is considered, $Y$ is placed in the range of $F(-, Z)$, a contradiction. Thus, for each $Z, A(Z)$ is equal to the range of $F(-, Z)$.

\subsection{Constructing a solution}

THEOREM 2.3. There is a set $U$ of reals such that for every uncountable analytic set $A$, there is a continuous function $f$ which maps $U$ bijectively to $A$.

Proof. We permute the logic from our heuristic construction of $F$. When we defined $F$, we fixed the domain $2^{\omega}$ in advance, and then specified a family of functions $F(-, Z)$ by transfinite recursion on that domain. Here, we define a family of continuous functions $f(-, Z)$ in advance. We then define a set $U$ of reals by transfinite recursion, on which those functions will be defined. We ensure that $f(-, Z)$ has the same value on the $\alpha$ th element of $U$ that $F(-, Z)$ has on $\alpha$.

Decomposing analytic sets. Suppose that $A$ is an analytic set. For each finite sequence $\sigma$, let $i(\sigma)$ denote the interval of reals in ${ }^{\omega} \omega$ which extend $\sigma$. Clearly, $i(\sigma) \cap A$ is analytic and therefore either $i(\sigma) \cap A$ is countable or it contains a perfect set. Since there are only countably many sequences $\sigma$, there are only countably many reals in the union of those sets $i(\sigma) \cap A$ for which $i(\sigma) \cap A$ is countable. Consequently, $A$ can be written as the union of 
a countable set $A_{\mathrm{c}}$ and an analytic set $A_{\mathrm{u}}$ such that for all $\sigma$, if $i(\sigma) \cap A_{\mathrm{u}}$ is nonempty, then it contains a perfect set. It is a technical point, but if $A$ is uncountable, then there are $A_{\mathrm{c}}$ and $A_{\mathrm{u}}$ as above such that $A_{\mathrm{c}}$ is countable and infinite.

Henceforth, we will consider only representations of analytic sets $A(Z)$ in the form $A_{\mathrm{c}}(Z) \cup A_{\mathrm{u}}(Z)$ as above. By invoking the Perfect Set Theorem, we will also assume that each analytic set comes equipped with the system of perfect trees

$$
\mathcal{T}(Z)=\left\{T(\sigma, Z): i(\sigma) \cap A_{\mathrm{u}}(Z) \neq \emptyset\right\}
$$

with the following properties: For each $T(\sigma, Z)$ in $\mathcal{T}(Z),[T(\sigma, Z)]$ is a subset of $i(\sigma) \cap A_{\mathrm{u}}(Z)$, and for unequal $\sigma$ and $\tau,[T(\sigma, Z)] \cap[T(\tau, Z)]=\emptyset$.

$A$ one-parameter family of continuous functions. We decompose ${ }^{\omega} \omega$ into $\left({ }^{\omega} \omega\right)_{0}$, those reals whose first element is 0 , and $\left({ }^{\omega} \omega\right)_{1}$, those reals whose first element is 1 . Our set $U$ will be the union of two disjoint sets, $U_{\mathbf{c}} \subset\left({ }^{\omega} \omega\right)_{0}$ and $U_{\mathrm{u}} \subset\left({ }^{\omega} \omega\right)_{1}$, so that our functions $f(-, Z)$ can continuously distinguish potential elements of $U_{\mathrm{c}}$ from potential elements of $U_{\mathrm{u}}$ in the following.

We define $U_{\mathrm{c}}$ to be the countable subset of $\left({ }^{\omega} \omega\right)_{0}$ consisting of those reals whose first element is 0 , whose second element is arbitrary, and whose remaining elements are all equal to 0 . For $(0, n, 0, \ldots) \in U_{\mathrm{c}}$, we define $f((0, n, 0, \ldots), Z)$ to be the $n$th element of $A_{\mathrm{c}}(Z)$.

We interpret the elements of $\left({ }^{\omega} \omega\right)_{1}$ as triples $\left(Y^{*}, Z^{*}, D^{*}\right): Y^{*}$ and $Z^{*}$ are viewed as having roles as in the definition of $F$ in Section 2.2; when evaluating $f(-, Z)$ on $U_{\mathrm{u}}$, if necessary $D^{*}$ is used to pick a real not yet in the range of $f(-, Z)$.

On $\left({ }^{\omega} \omega\right)_{1}$, we define $f$ by recursion on $\omega$. If the recursion reaches step $n$ without terminating, then we may assume that $f\left(\left(Y^{*}, Z^{*}, D^{*}\right), Z\right)\lceil n$ is equal to $Y^{*}\lceil n$ and we proceed as follows:

- If $Z\left\lceil n+1=Z^{*}\left\lceil n+1\right.\right.$ and $A_{\mathrm{u}}(Z) \cap i\left(Y^{*}\lceil n+1) \neq \emptyset\right.$, then let $f\left(\left(Y^{*}, Z^{*}, D^{*}\right), Z\right)(n)=Y^{*}(n)$.

- Otherwise, end the recursion and let $f\left(\left(Y^{*}, Z^{*}, D^{*}\right), Z\right)$ be the path through $T\left(i\left(Y^{*} \mid n\right), Z\right)$ canonically determined by $D^{*}$.

In short, while it appears that $Z$ is equal to $Z^{*}$ and that $Y^{*} \in A_{\mathrm{u}}(Z)$, we let $f\left(\left(Y^{*}, Z^{*}, D^{*}\right), Z\right)$ copy $Y^{*}$. Should this condition no longer hold, we use $D^{*}$ to pick an element in one of the perfect subsets of $A_{\mathrm{u}}(Z)$.

Defining $U_{\mathrm{u}}$. We define $U_{\mathrm{u}}$ to be the union of the sets $U_{\alpha}$ which appear in the following transfinite recursion. For $\alpha$ less than $2^{\omega}$, let $U_{\alpha}$ be the set of reals $\left\{\left(Y_{\beta}, Z_{\beta}, D_{\beta}\right): \beta<\alpha\right\}$. (In particular, $U_{0}=\emptyset$.) Let $\left(Y_{\alpha}, Z_{\alpha}\right)$ be the $<$-least pair $(Y, Z)$ such that $Y$ is not in the range of $f(-, Z)$ applied to $U_{\alpha}$ and $Y \in A_{\mathrm{u}}(Z)$. Let $D_{\alpha}$ be the least real satisfying the following two conditions on $D$ : first, for all $\beta$ less than $\alpha, D$ is not equal to $D_{\beta}$; second, 
for all $\beta$ less than $\alpha$ and all of the trees $T\left(\sigma, Z_{\beta}\right)$ in $\mathcal{T}\left(Z_{\beta}\right), D$ is unequal to $D_{\beta}$ and the path through $T\left(\sigma, Z_{\beta}\right)$ canonically determined by $D$ is not equal to $f\left(\left(Y_{\beta}, Z_{\beta}, D_{\beta}\right), Z_{\beta}\right)$. Then let $\left(Y_{\alpha}, Z_{\alpha}, D_{\alpha}\right)$ be the element of $\left({ }^{\omega} \omega\right)_{1}$ which represents this triple.

Here is an argument that $D_{\alpha}$ is well defined. The constraint that for each $\beta<\alpha, D_{\alpha}$ not equal $D_{\beta}$ rules out $\alpha$ many possible values for $D_{\alpha}$. Suppose that $\beta$ is less than $\alpha$ and $T\left(\sigma, Z_{\beta}\right) \in \mathcal{T}\left(Z_{\beta}\right)$. Since each real determines a unique path through $T\left(\sigma, Z_{\beta}\right)$, at most one real can determine the path $f\left(\left(Y_{\beta}, Z_{\beta}, D_{\beta}\right), Z_{\beta}\right)$ through $T\left(\sigma, Z_{\beta}\right)$. Further, the elements of $\mathcal{T}\left(Z_{\beta}\right)$ have disjoint sets of paths, so there is at most one real that can determine the path $f\left(\left(Y_{\beta}, Z_{\beta}, D_{\beta}\right), Z_{\beta}\right)$ through any element of $\mathcal{T}\left(Z_{\beta}\right)$. Thus, at most $\alpha$ many additional values are ruled out by the second constraint on $D_{\alpha}$. Since $\alpha$ is less than $2^{\omega}$, the set of values which are ruled out for $D_{\alpha}$ by either of the two cases has cardinality less than $2^{\omega}$, and $D_{\alpha}$ is well defined.

Verifying the properties of $U$. Suppose that $A(Z)$ is an uncountable analytic set.

The proof in Section 2.2 that there cannot be a least $\left(Y^{*}, Z^{*}\right)$ such that $Y^{*}$ is in $A\left(Z^{*}\right)$ but not in the range of $f\left(-, Z^{*}\right)$ on $U$, applies to $f(-, Z)$ exactly as it did to $F(-, Z)$. Consequently, $f(-, Z)$ maps $U$ onto $A(Z)$.

It only remains to verify that $f(-, Z)$ is injective on $U$. Clearly, $f(-, Z)$ is injective on $U_{\mathrm{c}}$ and maps $U_{\mathrm{u}}$ to $A_{\mathrm{u}}(Z)$, so we need only consider the possibility that two elements of $U_{\mathrm{u}}$ have the same image. Suppose that $\beta<\alpha$ and $f\left(\left(Y_{\beta}, Z_{\beta}, D_{\beta}\right), Z\right)=f\left(\left(Y_{\alpha}, Z_{\alpha}, D_{\alpha}\right), Z\right)$. It cannot be the case that $f\left(\left(Y_{\alpha}, Z_{\alpha}, D_{\alpha}\right), Z\right)$ is evaluated by staying in the first case of the recursion during each stage: in this case, $Z$ would equal $Z_{\alpha} ; f\left(\left(Y_{\alpha}, Z_{\alpha}, D_{\alpha}\right), Z\right)$ would equal $Y_{\alpha}$; by the choice of $Y_{\alpha}, Y_{\alpha}$ would not be in the range of $f\left(-, Z_{\alpha}\right)$ on earlier elements of $U_{\mathrm{u}}$, and that would be a contradiction. Thus, $f\left(\left(Y_{\alpha}, Z_{\alpha}, D_{\alpha}\right), Z\right)$ must be defined by using $D_{\alpha}$ to determine a path through one of the trees in $\mathcal{T}(Z)$. If $Z_{\beta}$ is not equal to $Z$, then $f\left(\left(Y_{\beta}, Z_{\beta}, D_{\beta}\right), Z\right)$ would also be a path through one of the trees in $\mathcal{T}(Z)$ and canonically determined by $D_{\beta}$. But different elements of $\mathcal{T}(Z)$ have disjoint sets of paths, so $f\left(\left(Y_{\beta}, Z_{\beta}, D_{\beta}\right), Z\right)$ and $f\left(\left(Y_{\alpha}, Z_{\alpha}, D_{\alpha}\right), Z\right)$ would have to be paths in the same tree. But this would contradict the fact that $D_{\beta}$ and $D_{\alpha}$ are not identical, since different reals canonically determine different paths in a perfect tree. The only remaining case is that $Z$ is equal to $Z_{\beta}$. But the choice of $D_{\alpha}$ precludes $f\left(\left(Y_{\alpha}, Z_{\alpha}, D_{\alpha}\right), Z_{\beta}\right)$ 's being equal to $f\left(\left(Y_{\beta}, Z_{\beta}, D_{\beta}\right), Z_{\beta}\right)$, so this case would also lead to a contradiction. Thus, $f(-, Z)$ is injective on $U$, which completes the proof of Theorem 2.3.

3. A question. Our argument in the previous section does not use any properties of the analytic sets other than their satisfying the Perfect Set Theorem and being parameterized by the reals. Consequently, it applies to 
the higher projective classes under suitable large cardinal assumptions. See [4] for more information on these.

Problem 3.1. Does there exist a projective set $U$ of reals such that for every uncountable analytic set $A$, there is a continuous function $f$ that maps $U$ bijectively to $A\left({ }^{1}\right)$ ?

\section{References}

[1] K. Gödel, The consistency of the axiom of choice and of the generalized continuum hypothesis, Proc. Nat. Acad. Sci. U.S.A. 24 (1938), 556-557.

[2] A. S. Kechris, Classical Descriptive Set Theory, Grad. Texts in Math. 156, Springer, 1995.

[3] N. N. Luzin, Leçons sur les ensembles analytiques, Gauthier-Villars, Paris, 1930.

[4] Y. N. Moschovakis, Descriptive Set Theory, Stud. Logic Found. Math. 100, NorthHolland, Amsterdam, 1980.

[5] W. Sierpiński, Problem 70, Fund. Math. 26 (1936), 334.

Department of Mathematics

University of California Berkeley

Berkeley, California 94720-3840

U.S.A.

E-mail: slaman@math.berkeley.edu

Received 20 April 1998

$\left({ }^{1}\right)$ Added in proof: Hjorth has shown that, yes, there is a projective set $U$ of reals such that for every uncountable analytic set $A$, there is a continuous function $f$ that maps $U$ bijectively to $A$. 